\title{
An Analysis of Leptonic and Semileptonic $B$ Decays
}

\section{Giovanni Banelli*}

Nikhef, Science Park 105, 1098 XG Amsterdam

E-mail: g.banelli@nikhef.nl

\begin{abstract}
We present a new strategy to explore New Physics contributions to leptonic and semileptonic $B$ decays. Using available measurements we put constraints on the Wilson coefficients of the usual model-independent low-energy effective Hamiltonian; general considerations about New Physics models are made, too. We devote particular attention to the effect of CP-violating phases of the short-distance coefficients, and provide predictions for the branching ratios that have not yet been measured.
\end{abstract}

The International Conference on B-Physics at Frontier Machines - BEAUTY2018

6-11 May, 2018

La Biodola, Elba Island, Italy

${ }^{*}$ Speaker. 


\section{Motivation}

The poster illustrates an example of a strategy that is more comprehensively discussed in [1].

Following the interest raised by the flavour anomalies $R_{K^{(*)}}$ and $R_{D^{(*)}}$ (see for instance [2] and references therein), a more profound investigation of the possibility of lepton flavour universality violation (LFUV) across different decay modes is necessary. In this work we explore $b \rightarrow u \ell \bar{v}_{\ell}$ transitions given by $B^{-} \rightarrow \ell^{-} \bar{v}_{\ell}$ and $\bar{B} \rightarrow \pi \ell^{-} \bar{v}_{\ell}$ meson decays. The first measurement of $\mathscr{B}\left(B^{-} \rightarrow\right.$ $\left.\mu^{-} \bar{v}_{\mu}\right)=(6.46 \pm 2.74) \times 10^{-7}[3]$, when compared with $\mathscr{B}\left(B^{-} \rightarrow \tau^{-} \bar{v}_{\tau}\right)$, can be used to test LFUV in leptonic decays. Moreover, leptonic decays are helicity suppressed in the SM whereas the same suppression can be lifted in the presence of scalar NP contributions. The same measurement quoted above, together with the semileptonic branching ratio $\mathscr{B}\left(\bar{B} \rightarrow \pi \ell^{-} \bar{v}_{\ell}\right)$ where $\ell$ denotes an average over electrons and muons, allows us to extract the magnitude of NP coefficients in a clean way. The upper bound on $\mathscr{B}\left(B^{-} \rightarrow e^{-} \bar{v}_{e}\right)$ will also help to constrain the allowed space for NP.

\section{Theoretical framework}

Our NP analysis uses the formalism of effective field theory. In this framework, the contributions to a given decay mode are described by a low-energy effective Hamiltonian containing Standard Model operators as well as contributions from physics beyond the SM:

$$
\mathscr{H}_{\mathrm{eff}}=\frac{4 G_{\mathrm{F}}}{\sqrt{2}} V_{u b}\left[\mathscr{O}_{V}^{\ell}+C_{S}^{\ell} \mathscr{O}_{S}^{\ell}+\ldots\right],
$$

where $\mathscr{O}_{V}=\left(\bar{u}_{L} \gamma^{\mu} b_{L}\right)\left(\bar{\tau}_{R} \gamma_{\mu} v_{\ell L}\right)$ is the Standard Model operator and $\mathscr{O}_{S}=\left(\bar{u}_{L} b_{R}\right)\left(\bar{\ell}_{R} v_{\ell L}\right)$ is an example of a dimension six effective NP operator, parametrised by the in general complex Wilson Coefficient $C_{S}^{\ell}$. In the same Hamiltonian, we can have contributions from other operators, which are discussed in [1]. Such a scalar operator is motivated by a popular extension of the Higgs sector, namely the Two Higgs Doublet Model (2HDM) as introduced in [4] and characterised by $C_{S}^{\mu}=C_{S}^{e} m_{\mu} / m_{e}=C_{S}^{\tau} m_{\mu} / m_{\tau}$. However the same strategy can also be applied to test the existence of a non-specified scalar particle, which for example can be assumed to interact only with the third lepton generation, i.e. $C_{S}^{e}=C_{S}^{\mu}=0$. Using the Hamiltonian Eq. 2.1 yields

$$
\mathscr{B}\left(B^{-} \rightarrow \ell^{-} \bar{v}_{\ell}\right)=\frac{G_{\mathrm{F}}^{2}}{8 \pi}\left|V_{u b}\right|^{2} f_{B^{-}}^{2} \tau_{B^{-}} M_{B^{-}}\left(1-\frac{m_{\ell}^{2}}{M_{B^{-}}^{2}}\right)\left|m_{\ell}+\frac{M_{B^{-}}^{2}}{\left(m_{b}+m_{u}\right)} C_{S}^{\ell}\right|^{2}
$$

where we observe that helicity suppression could indeed be lifted. In this expression, all hadronic physics is described by the decay constant $f_{B}$. On the other hand, semileptonic decays have a more involved expression described in [1] which includes transverse momentum dependent form factors like $f_{+}\left(q^{2}\right)$ and $f_{0}\left(q^{2}\right)$. The values of these hadronic parameters are currently determined using methods like lattice QCD and QCD sum rules [5].

\section{The strategy}

Our goal is to extract in the cleanest possible way the values of NP coefficients. Since the CKM matrix element $\left|V_{u b}\right|$ is determined assuming the SM, in order to control all NP effects, we 


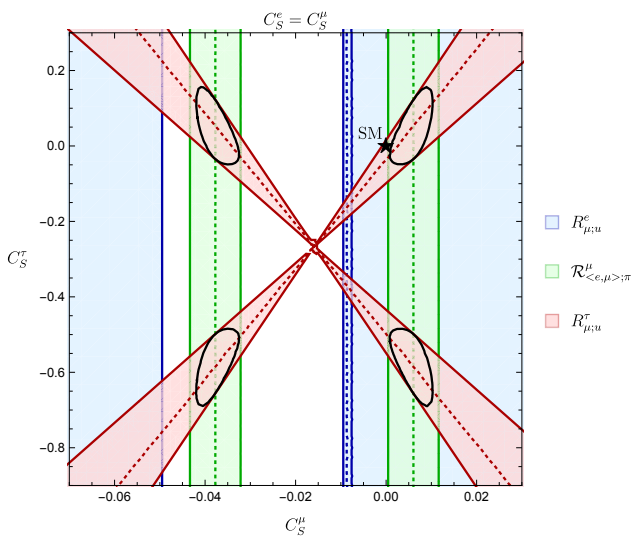

Figure 1: Allowed regions for the Wilson coefficients $C_{S}^{\mu}$ and $C_{S}^{\tau}$. The black contour represents a $1 \sigma$ fit to $R_{\mu ; u}^{\tau}$ and $\mathscr{R}_{\langle e, \mu\rangle ; \pi}^{\mu}$; the star indicates the SM solution.

introduce

$$
R_{\ell_{2} ; u}^{\ell_{1}} \equiv \frac{m_{\ell_{2}}^{2}}{m_{\ell_{1}}^{2}}\left(\frac{M_{B^{-}}^{2}-m_{\ell_{2}}^{2}}{M_{B^{-}}^{2}-m_{\ell_{1}}^{2}}\right)^{2} \frac{\mathscr{B}\left(B^{-} \rightarrow \ell_{1}^{-} \bar{v}_{\ell_{1}}\right)}{\mathscr{B}\left(B^{-} \rightarrow \ell_{2}^{-} \bar{v}_{\ell_{2}}\right)}
$$

for the leptonic decays, and

$$
\mathscr{R}_{\ell_{2} ; \pi}^{\ell_{1}} \equiv \frac{\mathscr{B}\left(B^{-} \rightarrow \ell_{1}^{-} \bar{v}_{\ell_{1}}\right)}{\mathscr{B}\left(\bar{B} \rightarrow \pi \ell_{2}^{-} \bar{v}_{\ell_{2}}\right)}, \quad \mathscr{R}_{\ell_{2} ; \pi}^{\ell_{1} ; \pi} \equiv \frac{\mathscr{B}\left(\bar{B} \rightarrow \pi \ell_{1}^{-} \bar{v}_{\ell_{1}}\right)}{\mathscr{B}\left(\bar{B} \rightarrow \pi \ell_{2}^{-} \bar{v}_{\ell_{2}}\right)}
$$

for the semileptonic modes, where $\left|V_{u b}\right|$ cancels. Unfortunately, for $\mathscr{B}\left(\bar{B} \rightarrow \pi \ell^{-} \bar{v}_{\ell}\right)$, only measurements averaged over electrons and muons are available. Consequently, in order to constrain $C_{S}^{\mu}$, we need to assume a relation between $C_{S}^{e}$ and $C_{S}^{\mu}$.

The simplest assumption we can make is electron-muon universality, $C_{S}^{e}=C_{S}^{\mu}$. Additionally, for the moment, we assume real coefficients. Scanning the parameter space for the NP coefficients results in the regions in Fig. 1: of the two regions allowed by our three constraints, one is compatible with the SM.

Next we convert the Wilson coefficients into predictions for other observables that have not yet been measured, $\mathscr{B}\left(B^{-} \rightarrow e^{-} \bar{v}_{e}\right)$ and $\mathscr{B}\left(\bar{B} \rightarrow \pi \tau^{-} \bar{v}_{\tau}\right)$. They are obtained by evaluating the $\left|V_{u b}\right|$-independent ratios $R_{\tau ; u}^{e}$ and $\mathscr{R}_{\langle e, \mu\rangle ; \pi}^{\tau ;} \quad \pi$ with the extracted values of the Wilson coefficients $\left(C_{e}\right.$ is of course dependent on the assumption made), and multiplying them with the measured $\mathscr{B}\left(B^{-} \rightarrow\right.$ $\left.\tau^{-} \bar{v}_{\tau}\right)$ and $\mathscr{B}\left(\bar{B} \rightarrow \pi \ell^{-} \bar{v}_{\ell}\right)$ branching ratios, respectively.

As mentioned before, we considered also different assumptions for the NP coefficients like the $2 \mathrm{HDM}$ and third generation-only scalar NP. However, regarding $\mathscr{B}\left(B^{-} \rightarrow e^{-} \bar{v}_{e}\right)$, which is very sensitive to scalar NP operators as they lift the helicity suppression, a noticeable enhancement is given only in the scenario of lepton flavour universal NP, where within one sigma it can reach as much as $10^{-7}$, i.e. an enhancement of $10^{5}$ with respect to the SM. As for $\mathscr{B}\left(\bar{B} \rightarrow \pi \tau^{-} \bar{v}_{\tau}\right)$, different models with their solutions give different predictions that cover the whole range between the SM expectation and the current upper bound. Consequently, better experimental measurements would be needed to discriminate between models once $\mathscr{B}\left(\bar{B} \rightarrow \pi \tau^{-} \bar{v}_{\tau}\right)$ is observed.

Finally we relax the assumption about real coefficients, and generalise them as $C_{S}^{\ell}=\left|C_{S}^{\ell}\right| e^{i \phi_{S}^{\ell}}$. We would like to explore the impact of these phases on our strategy. Noting that Fig. 1 corresponds 

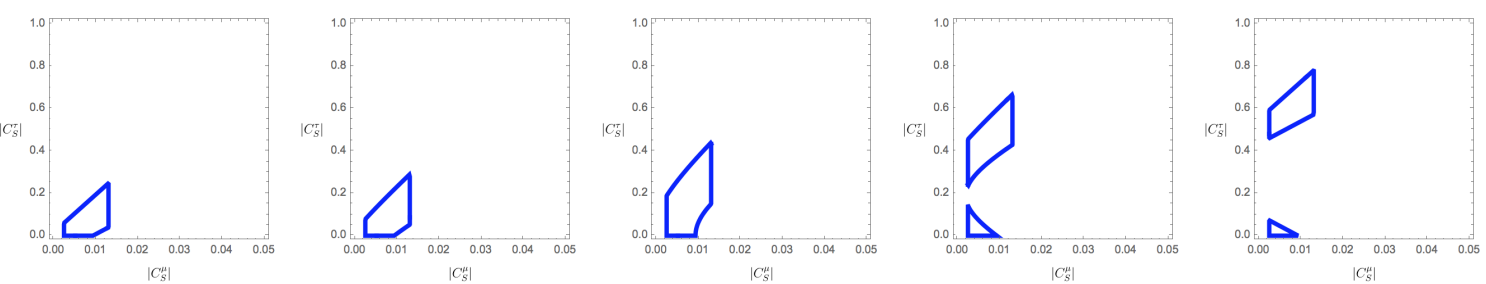

Figure 2: Allowed regions in the $\left|C_{S}^{\mu}\right|-\left|C_{S}^{\tau}\right|$ plane increasing $\phi_{S}^{\tau}$ in steps of $45^{\circ}$ from $0^{\circ}$ to $180^{\circ}$ from left to right, for $\phi_{S}^{\mu}=0^{\circ}$, and assuming electron-muon universality.

to phases $0^{\circ}$ and $180^{\circ}$, we will now include solutions for intermediate phases. As an example, we change the phase of $C_{\tau}$ between $0^{\circ}$ and $180^{\circ}$ in steps of $45^{\circ}$, giving the behaviour shown in Fig. 2. We observe that when no information on the phases of the NP coefficients is available, the space of the solutions for the magnitudes of the coefficients gets noticeably smeared. This demonstrates the strong impact of CP-violating phases on NP analyses.

\section{Conclusions}

We present a new strategy to probe NP effects in $b \rightarrow u \ell \bar{v}_{\ell}$ transitions. In the case of scalar NP we find consistency with the SM but also room for NP. Specific measurements for different lepton flavours, as well as higher precision will allow us to test compatibility with different NP models. We remark that nevertheless the possibility of new CP-violating phases represents a relevant obstacle in the determination of NP.

A similar strategy could be applied for $B_{c}$ decays and the related $b \rightarrow c$ transitions.

\section{Acknowledgments}

The author is grateful to Prof. Robert Fleischer, who supervised this master thesis research, Gilberto Tetlalmatzi-Xolocotzi and Ruben Jaarsma, the other two members of the group that helped the author in the work, and acknowledges the "Volkert van der Willigen" grant of the University of Amsterdam, which made the participation in this conference possible.

\section{References}

[1] G. Banelli, R. Fleischer, R. Jaarsma, G. Tetlalmatzi-Xolocotzi, in preparation.

[2] D. Bečirević, I. Doršner, S. Fajfer, N. Košnik, D. A. Faroughy, O. Sumensari, Scalar leptoquarks from GUT to accommodate the B-physics anomalies, hep-ph/1806.05689.

[3] A. Sibidanov et al. [Belle Collaboration], Search for $B^{-} \rightarrow \mu^{-} \bar{v}_{\mu}$ Decays at the Belle Experiment, Phys. Rev. Lett. 121 (2018) no.3, 031801 [hep-ex/1712.04123].

[4] W. S. Hou, Enhanced charged Higgs boson effects in $B^{-} \rightarrow \tau \bar{v}, \mu \bar{v}$ and $b \rightarrow \tau \bar{v}+X$, Phys. Rev. $D 48$ (1993), 2342.

[5] A. Khodjamirian, T. Mannel, N. Offen, Y. M. Wang, $B \rightarrow \pi \ell v_{l}$ Width and $\left|V_{u b}\right|$ from QCD Light-Cone Sum Rules, Phys. Rev. D 83 (2011), 094031 [hep-ph/1103.2655]. 